\title{
Stereoselective hydroxylation of an achiral cyclopentanecarboxylic acid derivative using engineered P450s BM-3†
}

\author{
Dieter F. Münzer, ${ }^{a}$ Peter Meinhold, ${ }^{b}$ Matthew W. Peters, ${ }^{b}$ Sabine Feichtenhofer,${ }^{c}$ Herfried Griengl, ${ }^{a}$ \\ Frances H. Arnold, ${ }^{* b}$ Anton Glieder ${ }^{c}$ and Anna de Raadt ${ }^{* a}$
}

Received (in Cambridge, UK) 2nd February 2005, Accepted 21st March 2005

First published as an Advance Article on the web 6th April 2005

DOI: $10.1039 / \mathrm{b501527h}$

\begin{abstract}
Substrate engineered, achiral carboxylic acid derivative 2 was biohydroxylated with various mutants of cytochrome P450 BM-3 to give two out of the four possible diastereoisomers in high de and ee. The BM-3 mutants exhibit up to 9200 total turnovers for hydroxylation of the engineered substrate, which without the protecting group is not transformed by this enzyme.
\end{abstract}

Biotransformations are restricted to compounds that are enzyme substrates. If a target molecule cannot be directly converted by an enzyme, or if the regio- and/or enantioselectivity of the reaction is low, at least two strategies can be employed to rectify the situation. First, given an enzyme system, directed evolution can be exploited to improve the synthetic outcome. ${ }^{1,2}$ Alternatively, the substrate can be engineered to improve its reactivity in a biotransformation, as in the docking/protecting (d/p) group concept. ${ }^{3,4}$ Both approaches have been developed to improve the applicability of bioconversions in chemical synthesis, especially for preparing chiral molecules. In this report, we show how protein engineering and substrate engineering can be combined to improve the synthetic outcome of a biohydroxylation.

A method for direct biohydroxylation of cyclic carboxylic acids to produce preparatively valuable starting materials, such as carboxylic acid $\mathbf{1}$ to compound $\mathbf{4}$ (Scheme 1), would be a valuable addition to an organic chemist's synthetic "tool box". Carbovir (Fig. 1), for example, is a carbocyclic nucleoside potentially active against human HIV. ${ }^{5} 4$ would be a potentially useful precursor to this much needed medication. Unfortunately, finding whole cell systems capable of hydroxylating substances such as $\mathbf{1}$ has proven difficult, especially when a specific product stereoselectivity is

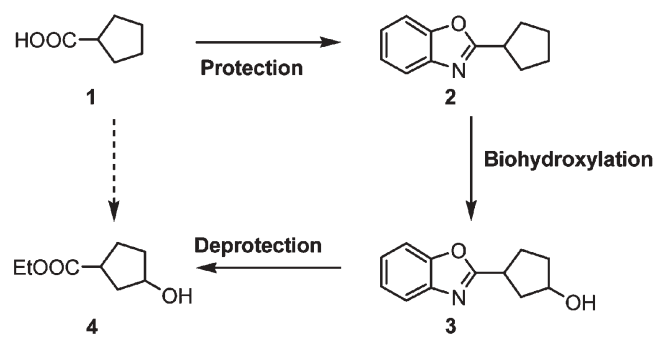

Scheme 1 The conversion of carboxylic acid 1 to product $\mathbf{4}$ employing the docking/protecting group concept.

$\dagger$ Electronic supplementary information (ESI) available: experimental details. See http://www.rsc.org/suppdata/cc/b5/b501527h/

*frances@cheme.caltech.edu (Frances H. Arnold)

deraadt@orgc.tu-graz.ac.at (Anna de Raadt) required. In addition, these simple carboxylic acids are troublesome to purify from complex fermentation/biotransformation mixtures due to their polarity and lack of UV absorbance. ${ }^{4}$

The $\mathrm{d} / \mathrm{p}$ group concept was found to circumvent these problems, at least in part. Carboxylic acid $\mathbf{1}$ was chemically modified to give model compound 2-cyclopentylbenzoxazole 2 (Scheme 1). This gave a substrate which was easy to manipulate as well as amenable to biohydroxylation with whole cell systems such as Cunninghamella blakesleeana DSM 1906. ${ }^{6-8}$ Protecting group removal provided end product 4 . Regardless of the microorganisms and fermentation conditions used, however, only a single diastereoisomer, $(S, S)-\mathbf{4}$, could be obtained with synthetically useful diastereoselectivities. ${ }^{6-8}$ Side products were also formed during this transformation because the microorganisms used catalyzed further, undesired transformations, such as oxidation. Consequently, a new tactic was sought.

Cytochrome P450 BM-3, a medium chain $\left(\mathrm{C}_{12}-\mathrm{C}_{18}\right)$ fatty acid hydroxylase from Bacillus megaterium, has been engineered to accept non-natural substrates with enhanced regioselectivity, enantioselectivity, catalytic rates and total turnover. Mutations in the active site, for example, enable the enzyme to hydroxylate alicyclic, heterocyclic, aromatic and even polyaromatic compounds. ${ }^{9-12}$ In one of our laboratories (FHA), P450 BM-3 has been engineered for efficient regio- and enantioselective alkane hydroxylation and alkene epoxidation. ${ }^{13-17} \mathrm{We}$ decided to test these mutants for their ability to hydroxylate model compound $\mathbf{2}$.

Whole-cell biotransformations were performed using E. coli DH5 $\alpha$ cells transformed with plasmids carrying the genes of 13 different P450 mutants (see supplementary material†). Results for the wild type and three mutants are listed in Table 1; the enantioselectivies obtained using the remaining ten mutants were similar to $139-3$.

Biohydroxylation using the wild type enzyme in $E$. coli gave very low enantioselectivity $(1.5 \%, S, S)$ and high diastereoselectivity $(87 \%)$. Mutant $139-3$ also afforded $(S, S)-3$, but in high ee $(79 \%)$ and de $(96 \%)$. Mutant B gave comparable selectivities to 139-3. In dramatic contrast, mutant $1-12 \mathrm{G}$ was found to produce the highly sought $(R, R)-3$ in high selectivities ( $89 \%$ ee, $94 \%$ de). Although the isolated yields of 3 were modest, ranging from $0.4 \%$ to $15 \%$, these

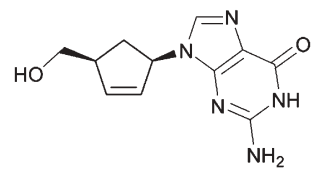

Fig. 1 Structure of (-)-carbovir. 
Table 1 Biohydroxylation of 2 with whole cell P450 BM-3 systems ${ }^{a}$

\begin{tabular}{llll} 
& & \\
Mutant & Main isomer 3 & ee & de \\
Wild type & $S, S$ & $1.5 \%$ & $87 \%$ \\
$139-3$ & $S, S$ & $79 \%$ & $96 \%$ \\
B & $S, S$ & $82 \%$ & $96 \%$ \\
$1-12 \mathrm{G}$ & $R, R$ & $89 \%$ & $94 \%$ \\
${ }^{a}$ Refer to the supplementary material for experimental details. \\
\hline
\end{tabular}

transformations have not been optimised with respect to such parameters as reaction conditions and host organism.

The enzymes from the four strains listed in Table 1 were then purified for in vitro enzymatic bioconversions. Results are summarised in Table 2.

Within experimental error, employing the purified enzymes produced results comparable to those in whole cell reactions. However, pure wild type BM-3 produced two new results: 3 slightly favored in the $(R, R)$ configuration and an unknown compound, tentatively identified as a product hydroxylated in the 2 position of the cyclopentane ring, as the major product ( $\sim 80 \%$, see supplementary material $\dagger$ ).

Total turnover numbers ( $\mathrm{ttn}$ ) and rates of formation of $\mathbf{3}$ were measured (Table 3). To determine ttn, the enzymes were diluted to a concentration at which the P450 is neither oxygen-limited nor inactivated due to dialysis of the flavins, a likely inactivation mechanism at low P450 concentrations. ${ }^{18}$ The three mutant enzymes catalyse the reaction at approximately the same rate, which is two orders of magnitude greater than that of the wild type. The total turnover number varies, however. Mutant B

Table 2 Biohydroxylation of $\mathbf{2}$ with purified enzymes ${ }^{a}$

\begin{tabular}{lllll}
\hline Mutant & $\% \mathbf{3}^{b}$ & Main isomer & ee & de \\
\hline Wild type & 20.0 & $R, R$ & 25.3 & n.d. $^{c}$ \\
139-3 & 85.9 & $S, S$ & 84.5 & 93.6 \\
B & 89.6 & $S, S$ & 87.9 & 96.4 \\
1-12G & 97.6 & $R, R$ & 87.7 & 95.3
\end{tabular}

${ }^{a}$ Refer to the supplementary material for experimental details. ${ }^{b}$ Proportion of $\mathbf{3}$ with respect to total products as determined with HPLC. ${ }^{c} \mathrm{Wt}$ protein did not yield sufficient product to accurately determine de of $\mathbf{3}$.

Table 3 Rates and total turnover numbers (ttn) of P450 BM-3 catalyzed hydroxylation reactions $^{a}$

\begin{tabular}{lll}
\hline Mutant & $\operatorname{ttn}^{b}$ & Rate $^{c}$ \\
\hline Wt & $1260 \pm 130$ & $2.6 \pm 0.3$ \\
$139-3$ & $3970 \pm 1080$ & $231 \pm 19$ \\
B & $9200 \pm 2040$ & $215 \pm 4$ \\
$1-12 G$ & $6840 \pm 1970$ & $213 \pm 14$
\end{tabular}

${ }^{a}$ Refer to the supplementary material for experimental details. ${ }^{b} \mathrm{ttn}$ is reported as nmol $\mathbf{3}$ per nmol protein. th values are reported instead of yields because reaction conditions with the whole cell experiments were not optimised. The protein is inactivated after 12 hours of incubation. ${ }^{c}$ Rates (nmol 3 per nmol protein per min) were determined by measuring formation of $\mathbf{3}$ over one minute. catalyses about an order of magnitude more turnovers than wild type.

The P450 BM-3 mutants examined in this work have at least 9 amino acid substitutions, including at least one active site mutation (A78V) (see supplementary material†). With linear alkanes such as octane, these mutations increase the regioselectivity of subterminal hydroxylation. ${ }^{14,16}$ Mutant $1-12 \mathrm{G}$ has the highest regioselectivity with linear alkanes ${ }^{16}$ and also exhibits the highest regioselectivity with substrate $2(97.6 \%)$. Although the mutants were engineered for the hydroxylation of short, linear alkanes, and not for substrates such as compound 2, ee's and de's of almost $90 \%$ were obtained. The fact that $1-12 \mathrm{G}$ produces the $(R, R)$ diastereomer of $\mathbf{3}$ is notable. This enzyme had previously been shown to catalyse the hydroxylation of octane primarily to $R$-2-octanol ( $39 \%$ ee), while all the other mutants produce mainly $S$-2-octanol (up to $58 \%$ ee). ${ }^{16}$ In addition to the active site mutation V78A, $1-12 \mathrm{G}$ contains mutations A82L and A328V. Only in this particular combination do these mutations generate $R$-2-octanol and $R, R-3$ from octane and 2, respectively. Consequently, this mutant favors a different binding conformation of the prochiral molecule $\mathbf{2}$ in which the pentyl ring is flipped by $180^{\circ}$ relative to the conformation in all the other mutants.

Wild type P450 BM-3 and the most promising mutants therefrom (139-3, B and L) were found to be inactive towards cyclopentanecarboxylic acid 1. Employing substrate engineered, achiral 2, however, enabled the biohydroxylation, and two out of the four possible isomers of $\mathbf{3}$ were prepared in high stereoselectivities. This result endorses the use of substrate engineering to broaden the range of compounds accepted by P450 enzymes. It also further demonstrates the functional flexibility of P450 BM-3 and supports the notion that the P450 BM-3 can be regarded as a "one-enzyme-fits-all" oxidation catalyst. ${ }^{16}$

We envisage that total turnover numbers, yields and stereoselectivities can be further improved by directly screening BM-3 mutant libraries with substrate 2 . Looking further into the future, it may also be possible to develop strains which produce the two remaining diastereoisomers of $\mathbf{3}$. Indeed, we predict that substrate engineering combined with protein engineering will enable new applications of biohydroxylation in organic synthesis.

Dieter F. Münzer, ${ }^{a}$ Peter Meinhold,${ }^{b}$ Matthew W. Peters, ${ }^{b}$ Sabine Feichtenhofer, ${ }^{c}$ Herfried Griengl, ${ }^{a}$ Frances H. Arnold, ${ }^{* b}$ Anton Glieder ${ }^{c}$ and Anna de Raadt*a

anstitut für Organische Chemie der Technischen Universität Graz, Stremayrgasse 16, A-8010, Graz, Austria.

E-mail: deraadt@orgc.tu-graz.ac.at; Fax: +43 316873 8740; Tel: +433168738751

${ }^{b}$ Division of Chemistry and Chemical Engineering, California Institute of Technology, Pasadena, CA 91125, USA.

E-mail: frances@cheme.caltech.edu; Fax: +1 626568 8743; Tel: +16263954162

${ }^{c}$ Research Centre Applied Biocatalysis, Institut für Molekulare Biotechnologie der Technischen Universität Graz, Petersgasse 14, A-8010, Graz, Austria.E-mail: a.glieder@tugraz.at;

Fax: 43316873 4071; Tel: 433168734074

\section{Notes and references}

1 K. E. Jaeger and T. Eggert, Curr. Opin. Biotechnol., 2004, 15, 305.

2 F. Valetti and G. Gilardi, Nat. Prod. Rep., 2004, 21, 490.

3 G. Braunegg, A. de Raadt, S. Feichtenhofer, H. Griengl, I. Kopper,

A. Lehmann and H. J. Weber, Angew. Chem. Int. Ed., 1999, 38, 2763.

4 A. de Raadt, H. Griengl and H. Weber, Chem. Eur. J., 2001, 7, 27. 
5 W. B. Parker, E. L. White, S. C. Shaddix, L. J. Ross, R. W. Buckheit, J. M. Germany, J. A. Secrist, R. Vince and W. M. Shannon, J. Biol. Chem., 1991, 266, 1754.

6 A. de Raadt, H. Griengl, M. Petsch, P. Plachota, N. Schoo, H. Weber, G. Braunegg, I. Kopper, M. Kreiner and A. Zeiser, Tetrahedron: Asymmetry, 1996, 7, 491.

7 A. de Raadt, H. Griengl, M. Petsch, P. Plachota, N. Schoo, H. Weber, G. Braunegg, I. Kopper, M. Kreiner, A. Zeiser and K. Kieslich, Tetrahedron: Asymmetry, 1996, 7, 467.

8 A. de Raadt, H. Griengl, M. Petsch, P. Plachota, N. Schoo, H. Weber, G. Braunegg, I. Kopper, M. Kreiner and K. Zeiser, Tetrahedron: Asymmetry, 1996, 7, 473.

9 D. Appel, S. Lutz-Wahl, P. Fischer, U. Schwaneberg and R. D. Schmid, J. Biotechnol., 2001, 88, 167.

10 Q. S. Li, J. Ogawa, R. D. Schmid and S. Shimizu, Appl. Environ. Microbiol., 2001, 67, 5735.
11 Q. S. Li, U. Schwaneberg, P. Fischer and R. D. Schmid, Chem. Eur. J., 2000, 6, 1531

12 A. B. Carmichael and L. L. Wong, Eur. J. Biochem., 2001, 268, 3117.

13 E. T. Farinas, U. Schwaneberg, A. Glieder and F. H. Arnold, $A d v$. Synth. Catal., 2001, 343, 601.

14 A. Glieder, E. T. Farinas and F. H. Arnold, Nat. Biotechnol., 2002, 20, 1135.

15 E. Farinas, T. M. Alcalde and F. H. Arnold, Tetrahedron: Asymmetry, 2003, 60, 525 .

16 M. W. Peters, P. Meinhold and F. H. Arnold, J. Am. Chem. Soc., 2003, 125, 13442 .

17 T. Kubo, M. W. Peters, P. Meinhold and F. H. Arnold, submitted, 2004.

18 P. R. de Montellano, Cytochrome P450: Structure, Mechanism, and Biochemistry, 2 edn., Plenum Press, New York, 1995. 